\title{
Perceptions of vocational trainees on gender and racial disadvantage within the Thames vocational training programme
}

\author{
K. Anees, ' R. Bedi, ${ }^{2}$ D. C. Rule, ${ }^{\dagger 3}$ and J. D. Lewsey, ${ }^{4}$
}

\begin{abstract}
Background A recent report has suggested that vocational trainees within London experienced racial or gender disadvantage during their selection. This exploratory study did not investigate the extent or the nature of this disadvantage.

Aim To undertake a survey using a pre-tested questionnaire with dental vocational trainees on the Thames Scheme. The questionnaire explored perceived and experienced aspects of gender and racial disadvantage during their vocational training programme.

Results 127 trainees completed the questionnaire (response rate 92\%). Minority ethnic respondents were more than twice as likely to feel their selection was influenced by gender (odds ratio $[\mathrm{OR}] 2.25,95 \%$ Confidence Interval [CI] 1.02, 5.10) and more than three times likely to feel selection was influenced by their race when compared with their white colleagues (OR 3.05, 95\%; CI 1.01,11.45). The majority of trainees did not perceive any disadvantage whilst on the vocational training course. For example, only five respondents (4\%) felt that minority ethnic individuals were treated less favourably during the vocational training course.
\end{abstract}

Conclusion In conclusion, this preliminary study has attempted to explore inter-ethnic differences within the profession on perceived racial disadvantage and possible strategies for change. It is clear that the perception of disadvantage is greater than the reality within the experience of most trainees.

A recent study by Bartlett et al. recorded the experience of undergraduates from three London dental schools and trainers from the South East of England on their vocational training (VT) programme. ${ }^{1}$ The findings highlighted the importance of the location of the trainee post to the prospective applicant and the experience of racial or gender disadvantage within the interview process. ${ }^{1}$ However, it was appar-

${ }^{1}$ Lecturer, Eastman Dental Institute for Oral Health Care Sciences, University of London, and Public Health Co-ordinator for the Muslim Council of Britain; ${ }^{2}$ Professor and Co-director, WHO

Collaborating Centre for Disability, Culture and Oral Health, National Centre for Transcultural Oral

Health, Eastman Dental Institute for Oral Health

Care Sciences, University of London; ${ }^{3 \dagger}$ (Deceased)

Dean of Postgraduate Dentistry, Thames

Postgraduate Medical \& Dental Education, 33

Millmon Street, London WC1N 3EJ; ${ }^{4}$ Research

Fellow, Bio-Statistics Unit, Eastman Dental Institute for Oral Health Care Sciences, University of London.

${ }^{\star}$ Correspondence to Professor Raman Bedi, National Centre for Transcultural Oral Health, Eastman

Dental Institute, University College London. 256

Gray's Inn Road. London WC1X 8LD.

email:R.Bedi@eastman.ucl..ac.uk

REFEREED PAPER

Received 13.11.00; Accepted 02.04.01

(C) British Dental Journal 2001; 191: 208-212 ent that the questions within the study were limited and the researchers themselves stated that there was a need for further

It has been estimated that approximately $8 \%$ of the NHS workforce are from minority ethnic backgrounds ${ }^{2}$ and it is perceived that these groups undergo racial disadvantage $^{3-5}$. Several government initiatives have also been launched to tackle racial discrimination within the NHS.6,7 This promotion of equal opportunities has gained further impetus from the recent publication of the McPherson Report into the tragic death of Stephen Lawrence. ${ }^{8}$ Whilst literature concerning disadvantage and health professionals within the UK medical press may be limited, this is an area that has been extensively explored within the US. ${ }^{9-12}$

The US views racial disadvantage in the form of proportionality, with disadvantage investigation of this important area. ${ }^{1}$ differences, which need to be explained. primarily being shown in terms of underrepresentation of any particular ethnic or racial group..$^{13}$ One national response, to improve the proportional representation of minority groups, has been the policy of 'affirmative action. ${ }^{14}$ The underlying conceptual model for this initiative is the belief that only by positive discrimination can any racial imbalance be rectified. One example is the active recruitment and appointment of under-represented medical staff in order to reflect the ethnic and racial mix of the society it serves ${ }^{14}$. The argument is further extended by the notion that by increasing minority representation within the medical profession this inevitably improves access and care for the minority populations.

The debate in the UK has focused less on the ethnic representation within the medical and dental professions in general, but more on adequate representation within positions of responsibility, such as consultant posts and senior management positions. ${ }^{15,16}$ Racial disadvantage has also been shown in employment studies, that is foreign doctors and UK graduated doctors from a minority ethnic background have greater difficulty in obtaining suitable training posts. ${ }^{17-20}$

The aim of this project was to record the perceptions and experiences of Thames region vocational trainees on racial $^{*}$ and gender $^{* *}$ disadvantage encountered during their selection and training in their VT scheme.

\section{Method}

The sample consisted of all 136 dentists registered on the North Thames Vocational Training Scheme in 1998. The support of the Dean of Postgraduate Dental Education for the Thames region and Vocational

${ }^{*}$ Racial differences relate to the group a person belongs to as a result of a mix of physical features (eg skin colour, hair texture), ancestry and geographical origins. Race has been traditionally considered as being defined by others whilst ethnicity is selfdefined. Thus the concept of ethnicity is one where an individual self-defines the group to which they belong as a result of cultural habits or beliefs and other factors such as language, religion, diet, etc. These differentials between ethnicity and race are being eroded and increasingly it is being recognized that they are so intertwined that consideration of one necessarily requires consideration of the other. Therefore, in this paper, race and ethnicity will be used as synonyms except where there are clear

${ }^{*}$ Gender is predominantly considered as it impacts upon minority ethnic groups. 


\begin{tabular}{|c|c|c|}
\hline Section & Question & Question asked and possible response \\
\hline A (personal details) & $\mathrm{I}-7$ & Age, sex, date of birth, ethnicity, religion, date and place of dental qualification. \\
\hline B (location) & $8-10$ & $\begin{array}{l}\text { Was the location of the job vital to your choice of practice? Yes/No } \\
\text { Did you want a job close to your dental school? Yes/No } \\
\text { Would you have been prepared to wait six months for the right location? Yes/No }\end{array}$ \\
\hline $\begin{array}{l}\mathrm{C} \text { (selection on to } \\
\text { and treatment } \\
\text { during VT) }\end{array}$ & $11-17$ & $\begin{array}{l}\text { Do you believe that the VT trainer was influenced by your gender, race (ethnicity) or both? } \\
\text { Gender/race (ethnic background)/Both/No influence of gender or race } \\
\text { Women are less favourably selected on to their VT course. Agree/Disagree } \\
\text { Women are less favourably treated during their VT course. Agree/Disagree } \\
\text { People from an ethnic minority background are less favourably selected onto their VT course. Agree/Disagree } \\
\text { People from an ethnic minority background are less favourably treated during their VT course. Agree/Disagree } \\
\text { Ethnic minority women are less favourably selected onto their VT course. Agree/Disagree } \\
\text { Ethnic minority women are less favourably treated during their VT course. Agree/Disagree }\end{array}$ \\
\hline $\begin{array}{l}\text { D (disadvantage } \\
\text { within the NHS) }\end{array}$ & $18-20$ & $\begin{array}{l}\text { Reports have highlighted that women have difficulties in developing a career in the NHS. Do you think this is } \\
\text { reflected in the dental profession? Yes/No } \\
\text { Reports have highlighted that ethnic minorities have difficulties in developing a career in the NHS. Do you think } \\
\text { this is reflected in the dental profession? Yes/No } \\
\text { Reports have highlighted that women of ethnic minority have difficulties in developing a career in the NHS. Do you } \\
\text { think this is reflected in the dental profession? Yes/No }\end{array}$ \\
\hline $\begin{array}{l}\text { E (strategies for } \\
\text { change) }\end{array}$ & $21-23$ & $\begin{array}{l}\text { Do you think that it is important for VT trainers to be trained in and have awareness of gender issues? Yes/No } \\
\text { Do you think that it is important for VT trainers to be trained in and have awareness of cultural/religious issues? Yes/No } \\
\text { Do you think the national VT scheme should allocate a number of reserved places to help those who have } \\
\text { difficulties in mobility due to: } \\
\text { gender reasons? Yes/No } \\
\text { cultural reasons? Yes/No } \\
\text { religious reasons? Yes/No }\end{array}$ \\
\hline
\end{tabular}

Training area coordinators was obtained. Qualitative and quantitative components of the study design were developed with the Thames Region education advisor.

\section{Questionnaire development}

The questionnaire was developed and tested using two focus group meetings, each group consisting of nine qualified dentists per group. Through discussion in both group meetings, the outline of the questionnaire was ascertained. Before distribution of the questionnaire a pilot study was conducted amongst a group of dental postgraduate students.

The questionnaire consisted of five prominent domains (Table 1). Firstly, questions regarding personal details (including age, gender, ethnic background, religious affiliation, etc) were included. Secondly, their experiences and attitudes to the location of their VT post were asked for and thirdly, their perceptions and actual experiences of selection on to, and treatment during their VT were obtained. Also, the respondents perceived disadvantage of careers within the NHS was recorded and finally, their views on possible strategies for change within the VT scheme were asked for.

A list of trainees was obtained from the Thames Postgraduate Dean and a copy of the questionnaire and covering letter sent in a sealed envelope to each trainee. Anonymity was assured.

All questionnaires were completed and access to completed forms was restricted to only one of the research team. No follow up posting was undertaken for the nonrespondents.

\section{Statistical analysis}

Logistic regression was employed to examine differences by gender and ethnicity to responses to questions posed in sections C-E of the questionnaire (Table 1).

Statistical results are displayed as odds ratios (the exponential of regression estimates), a procedure which has widespread use in oral health research. The odds ratios reported are adjusted, ie odds ratios for the gender effect have the effect of ethnicity included in the model (whether significant or not) and vice-versa. The interaction effect between gender and ethnicity was also considered in the modelling process but left out of all final models due to both its lack of magnitude and significance. The sample size was not large enough to investigate the interaction between gender and ethnicity with statistical rigour. Statistical signifi- 


\begin{tabular}{|c|c|c|c|}
\hline Table 2 & \multicolumn{3}{|c|}{$\begin{array}{l}\text { Logistic regression of how VT trainees perceived bias during the selection } \\
\text { procedure on to the training scheme by gender and ethnic background }\end{array}$} \\
\hline \multirow{2}{*}{\multicolumn{2}{|c|}{ Selection procedure questions }} & \multicolumn{2}{|c|}{ Covariates } \\
\hline & & Gender & Ethnicity \\
\hline \multicolumn{2}{|c|}{ Q12: Female selection } & $\begin{array}{l}\text { OR (females:males) } \\
4.25,95 \% \\
\text { Cl I.77-10.21 }\end{array}$ & $\begin{array}{l}\text { OR (minority ethnic:white) } \\
6.00,95 \% \\
\mathrm{Cl} 2.27-15.84\end{array}$ \\
\hline \multicolumn{2}{|c|}{ Q14: Minority ethnic selection } & NS & $\begin{array}{l}\text { OR } 2.48,95 \% \\
\text { Cl } 1.01-6.08\end{array}$ \\
\hline \multicolumn{2}{|c|}{ Q16: Minority ethnic female selection } & $\begin{array}{l}\text { OR } 2.08,95 \% \\
\text { Cl } 0.95-4.55\end{array}$ & $\begin{array}{l}\text { OR } 3.75,95 \% \\
\text { Cl 1.63-8.64 }\end{array}$ \\
\hline
\end{tabular}

$\mathrm{OR}$, odds ratio; $\mathrm{Cl}$, confidence interval; NS, not significant
(Table 2). However, it is worth noting that these differences were not reflected when respondents were asked about treatment of the previously mentioned groups during the VT course itself.

In fact, the majority of trainees did not perceive any disadvantage whilst on the VT course. For example, only five respondents (4\%) felt that minority ethnic individuals were treated less favourably.

Due to the modest sample size, potential interaction effects between gender and ethnicity could not be estimated with statistical rigour. However, there was some evidence to suggest that minority ethnic women responded differently from white respondents and minority ethnic men. The majority of minority ethnic women perceived disadvantage within the selection procedure whereas within other groups only a minority of respondents felt similarly.

The three subsequent questions dealt with perceived disadvantage for women, minority ethnic individuals and minority ethnic women establishing a career within the NHS. As can be seen in Table 3 there were no significant gender or ethnic effects when the question posed pertained to disadvantages for women. However, for the subsequent two questions significant effects were found although the gender effect for question 19 (Table 1) was not significant.

\section{Strategies for change}

The last three questions on the questionnaire deal with potential strategies for change, with the final question being split into three parts (Table 1, question 23). Strategy options were outlined on the questionnaire, which included training and awareness of gender and cultural/religious issues, as well as the possible allocation of places within VT schemes for gender, cultural or religious issues.

\section{Training in awareness issues}

Over 90 subjects (70\%) responded affirmatively to the statement, 'Do you think that it is important for vocational trainers to be trained in, and have awareness of, gender issues?' A significant difference was detected by ethnicity (OR 3.35, 95\%; CI 1.46-7.67) and gender (OR 2.05, 95\%; CI 0.9-4.7) with 


\begin{tabular}{|c|c|c|c|}
\hline Table 3 & \multicolumn{3}{|c|}{$\begin{array}{l}\text { Logistic regression of how VT trainees perceived disadvantage for women, } \\
\text { minority ethnic individuals and minority ethnic women establishing a } \\
\text { career within the NHS }\end{array}$} \\
\hline \multirow{2}{*}{\multicolumn{2}{|c|}{ NHS disadvantage questions }} & \multicolumn{2}{|c|}{ Covariates } \\
\hline & & Gender & Ethnicity \\
\hline \multicolumn{2}{|c|}{ Q18: Female disadvantage } & NS & NS \\
\hline \multicolumn{2}{|c|}{ Q19: Minority ethnic disadvantage } & NS & $\begin{array}{l}\text { OR (minority ethnic:white) } \\
4.14,95 \% \\
\text { Cl } 1.70-10.08\end{array}$ \\
\hline \multicolumn{2}{|c|}{ Q20: Minority ethnic female disadvantage } & $\begin{array}{l}\text { OR (female: male) } \\
2.5 I, 95 \% \\
\text { Cl I.15-5.49 }\end{array}$ & $\begin{array}{l}\text { OR } 3.45,95 \% \\
\text { Cl I.54-7.69 }\end{array}$ \\
\hline
\end{tabular}

NS, not significant the ethnic effect being greater than the gender effect.

The gender CI value passes through 1 , and as such 'no effect' cannot be excluded, however, although strictly statistically significant it is on the borderline of significance.

Training in cultural and religious issues was deemed important by 78 (61\%) of subjects. A significant difference was detected with regard to ethnicity but not gender.

\section{Reservation of VT places for gender,} culture and religious background

Subjects were requested to answer yes or no for each of the sections in question 23 of the questionnaire (Table 1), and everyone responded to the question.

Ninety-nine respondents (78\%) felt that no reservation of places should be undertaken due to gender, culture or religious background. There were no significant gender differences on reservation of places due to gender, culture or religious background.

A significant ethnic effect is present for all categories, with minority ethnic respondents over 4.5 times more likely to agree with reservation of places on the basis of gender (OR 2.67, 95\%; CI 1.01, 7.25) and over 2.5 times on the basis of religion, than their white counterparts (OR 4.55, 95\%; CI $1.46,14.22)$.

\section{Discussion}

Exploration of ethnic and gender disadvantage is a sensitive task and from the outset it was determined that the number of questions used and the ranges of topics covered within the questionnaire would be limited to achieve maximum compliance. It is recognized that the potential for asking leading questions may have biased the results, and therefore care should be taken with the interpretation of the results. Certain shortcomings with regard to the question structure and content must be highlighted. The question concerning gender and/or race influences upon the trainer does not specify whether this 'influence' is negative or positive in nature, nor indeed whether the trainer is indeed the current trainer for the trainee, or a trainer by whom the trainee has been interviewed. These aspects need further clarification and drawing any conclusions from these findings must be approached carefully. However, the questionnaire had been finalized and pre-tested and its administration was undertaken with assistance from the Thames Region Vocational Training Office. The whole process was explained to the VT and regional advisors and their cooperation obtained. Although it is understood that the ethnicity and views of the vocational trainers may be an important factor, this aspect was considered beyond the scope of this study.
The present study confirms the perception that gender and ethnicity influenced selection on to VT courses and supported the findings recorded by Bartlett et al. ${ }^{1}$. Although care should be taken in comparing these two studies, they highlight some interesting findings. The choice of practice was not associated with the location of the dental school where the respondents qualified. The importance of the location of the employing dental practice appears to be sufficient to stop prospective trainees from starting their VT for at least six months. Twenty-one percent $(\mathrm{n}=29)$ of subjects compared to $37 \%(n=40)$ in the study by Bartlett et al. were prepared to defer the start of their VT by six months in order to find the right location. However, whether many did actually wait six months is uncertain. ${ }^{1}$ Whilst both studies highlight location as important, the reasons for this are not clear. The importance of location may reflect the preference or need for trainees to be closer to family, home and friends than the school from which they qualified. Further research is needed to determine the reasons for the importance of location to vocational trainees. In the Bartlett study $78 \%(\mathrm{n}=84)$ felt that gender or race had played a part in their selection, compared with $36 \%$ in this study. ${ }^{1}$ Results from both studies were irrespective of gender, but influenced by the respondents' ethnic background.

There is little documentation on disadvantage for women and minority ethnic groups within the selection procedure for vocational dental training. Most respondents believed that no disadvantage existed in the treatment of any of the trainees. The reasons for this may be many-fold but include the fact that any trainee working will have come through an interview with the trainer unlikely to select an individual with whom they do not think a rapport can be built. The professional nature of dentistry would also come into play with any 'less favourable' treatment being considered unprofessional. In addition, it may be useful in the future to look more closely at the trainers, particularly their ethnic make up, as this may have a bearing on the findings. To compare trainers from different areas where ethnic make up may be different 
would also be a useful comparison.

The questionnaire in this study asked respondents three questions on their perception of disadvantage within the NHS for women, minority ethnic groups and, more specifically, minority ethnic women. The results of this study seem to confirm the difference of opinion reflected within the literature with $56 \%(\mathrm{n}=70)$ of subjects feeling that women do not have difficulties developing a career within the NHS.

Minority ethnic subjects were significantly more likely to perceive that disadvantage within the NHS occurred with minority ethnic subjects and particularly minority ethnic women than with their white counterparts.

Reports within the literature concerning race and ethnic disadvantage need to be carefully and objectively studied. This study highlights the differing perceptions of white and minority ethnic subjects on the subject of disadvantage. It is not possible to determine if white subjects under-estimate or minority ethnic subjects over-estimate the extent of disadvantage. However, it is clear that there is a need for a greater awareness and understanding of race and gender issues.

This study does show that the perception of gender disadvantage is far reduced in comparison to ethnic disadvantage. Explorations of solutions and strategies for change were mixed, with regard to reserving places for certain groups, which showed marked, interethnic differences. The results could be interpreted as showing the strength of feeling within minority ethnic subjects with regard to the disadvantage they encounter.

In conclusion, this preliminary study has attempted to explore interethnic differences within the profession on perceived disadvantage and possible strategies for change. It is clear that the perception of disadvantage is greater than the reality within the experience of most trainees. However, it is clear that further and more detailed research should be undertaken to understand these important interethnic and gender views.

David C. Rule co-supervised this project and approved the final manuscript. He sadly passed away before seeing the publication of his hard work.

1. Bartlett D W,Coward P Y, Goodsman D, Darby J. Experience of undergraduates from three London dental schools and trainers from the south east of England on interviews for vocational training in 1996. Br Dent J 1997; 183: 284-288.

2. Department of Health. 'Frank Dobson Launches NHS Equal Opportunity Awards. 98/118. 30th March 1998

3. (anonymous) Trying to overcome racism in the NHS. Br Med J 2000; 320(7235): 657.

4. McKenzie K. Something borrowed from the blues? Br Med J 1999; 318: 616-617.

5. Carnall D. Circumventing racism in the NHS. Br Med J 1997; 314: 7081

6. Warden J. Consultants' merit award system to be reformed Br Med J 1998; 317: 429

7. Bedi R. Racial discrimination in distinction awards. NHS monitoring of discrimination should be more transparent. Br Med J 1998; 316(7149): 1977-1978

8. MacPherson W. The Stephen Lawrence Inquiry. London, HMSO, 1999.
9. Palepu A, Carr P L, Friedman R H, Amos H, Ash A S, Moskowitz M A. Minority faculty and academic rank in medicine. J Am Med Assoc 1998; 280(9): 767-771.

10. Petersdorf R G, Turner S K, Nickens H W, Ready T. Minorities in medicine: Past, present and future. Academic Med 1990; 65: 663-670.

11. Palepu A, Carr P L, Friedman R H, Ash A S, Moskowitz M A. Specialty choices, compensation, and career satisfaction of underrepresented minority faculty in academic medicine. Academic Med 2000; 75(2): 157-160

12. Kassebaum D G, Szenas P L. Factors influencing the speciality choices of 1993 medical school graduates. Academic Med 1994; 69: 164-170.

13. Tekian A. A thematic review of the literature on underrepresented minorities and medical training, 1981-1995: Securing the bridge to diversity. Academic Med 1997; 72:(supplement) 140-146.

14. Cohen J J. Finishing the bridge to diversity. Academic Med 1997; 72: 103-109.

15. Rajarayan R K. Employmment opportunities. In Bedi R, Bahl V, Rajarayan R. (eds) Dentists, Patients and Ethnic Minorities. (eds) London, Faculty of General Dental Practitioners (UK) Royal College of Surgeons, 1996

16. Esmail A, Everington S, Doyle H. Racial discrimination in the allocation of distinction awards? Analysis of list of award holders by type of award, speciality and region. $\mathrm{Br} \mathrm{Med} \mathrm{J}$ 1998; 316: 193-195

17. Percival G, Ali A. Ethnic minority doctors in general practice. London: Commission for Racial Equality (1994).

18. Sharma P. The role of ethnic monitoring in the planning of health care services. London: Commission for Racial Equality (1995).

19. McKeigue M P, Richards J D M, Richards P. Effects of discrimination by sex and race on the early careers of British medical graduates during 1981 to 1987. Br Med J 1990; 301: 961-964.

20. Esmail A, Everington S. Racial discrimination against doctors from ethnic minorities. Br Med J 1993; 306: 691-692. 